\title{
Long-Term Treatment with Apixaban in Patients with Atrial Fibrillation: Outcomes during the Open-Label Extension following AVERROES
}

\author{
Alexander P. Benz ${ }^{1}$ John W. Eikelboom ${ }^{1}$ Salim Yusuf ${ }^{1}$ Stefan H. Hohnloser ${ }^{2}$ Anja Kahl ${ }^{3}$ \\ Heather Beresh ${ }^{1}$ Kumar Balasubramanian ${ }^{1}$ Jeff S. Healey ${ }^{1}$ Stuart J. Connolly ${ }^{1}$ \\ ${ }^{1}$ Population Health Research Institute, Hamilton, Ontario, Canada \\ Address for correspondence Alexander P. Benz, MD, Population \\ ${ }^{2}$ Division Clinical Electrophysiology, Department of Cardiology, \\ Health Research Institute, 237 Barton St. E., Hamilton, ON, L8L 2X2, \\ Johann Wolfgang Goethe University, Frankfurt, Germany \\ ${ }^{3}$ Bristol-Myers Squibb, Lawrenceville, New Jersey, Unites States \\ Canada (e-mail: Alexander.Benz@phri.ca).
}

Thromb Haemost 2021;121:518-528.

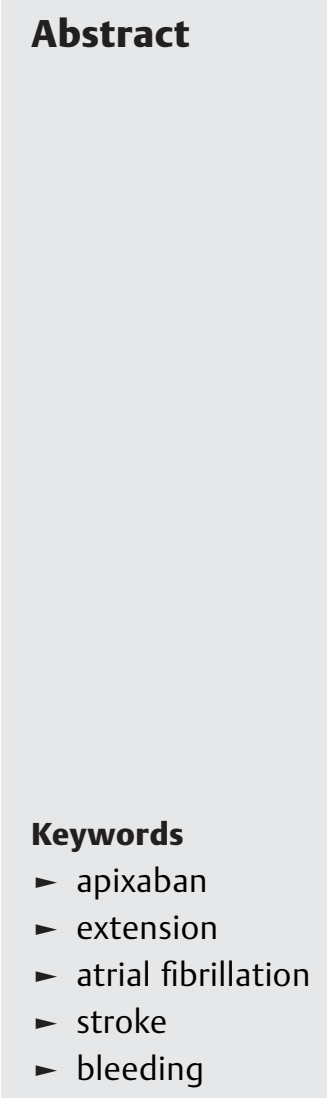

Background AVERROES, a randomized controlled trial in high-risk patients with atrial fibrillation, unsuitable for vitamin $\mathrm{K}$ antagonist therapy, demonstrated efficacy and safety of apixaban compared with aspirin. At the conclusion of the double-blind phase, an open-label extension was initiated to allow study participants to receive apixaban until it became locally available. This study reports outcomes of patients on apixaban during the open-label extension.

Methods Rates of stroke or systemic embolism, hemorrhagic stroke, major bleeding, and other outcomes during the open-label extension are reported.

Results Of the 5,599 participants enrolled in AVERROES, 3,275 (58.5\%) received apixaban during the open-label extension. Median (interquartile range) follow-up in the open-label extension was $3.0(2.5-3.5)$ years. The rate of stroke or systemic embolism during the open-label extension was $1.0 \%$ per year, and the annual rates of hemorrhagic stroke and major bleeding were 0.3 and $1.2 \%$, respectively. After adjustment for imbalances in patient variables, event rates in patients on apixaban during the openlabel extension were similar to those of patients receiving apixaban during AVERROES. Additional analyses in all patients who received apixaban, at any time from the start of AVERROES to the end of the open-label extension, were performed. This cohort $(n=4,414)$ showed annual event rates of $1.1 \%$ for stroke or systemic embolism, $0.3 \%$ for hemorrhagic stroke, and $1.2 \%$ for major bleeding.

Conclusion During the open-label extension, annual rates of stroke or systemic embolism, hemorrhagic stroke, and major bleeding remained as low as those observed during apixaban treatment in AVERROES. These data support the long-term efficacy and safety of apixaban in patients with atrial fibrillation. received

February 20, 2020

accepted after revision

August 18, 2020

published online

October 4, 2020
DOI https://doi.org/

$10.1055 / \mathrm{s}-0040-1717115$. ISSN 0340-6245. (c) 2020. The Author(s).

This is an open access article published by Thieme under the terms of the Creative Commons Attribution-NonDerivative-NonCommercial-License, permitting copying and reproduction so long as the original work is given appropriate credit. Contents may not be used for commercial purposes, or adapted, remixed, transformed or built upon. (https://creativecommons.org/ licenses/by-nc-nd/4.0/) Georg Thieme Verlag KG, Rüdigerstraße 14, 70469 Stuttgart, Germany 


\section{Introduction}

Atrial fibrillation is the most prevalent heart rhythm disorder affecting many millions of patients worldwide, ${ }^{1,2}$ and is associated with an increased risk for ischemic stroke. ${ }^{3,4}$ Oral anticoagulation is effective in reducing thromboembolic events in high-risk patients with atrial fibrillation, but carries an increased risk of bleeding. While treatment with vitamin $\mathrm{K}$ antagonists has been standard of care for decades, clinical practice in pharmacological prevention of thromboembolism in patients with atrial fibrillation has undergone a substantial transformation since the pivotal trials of direct oral anticoagulants were conducted. ${ }^{5-7}$

AVERROES was a double-blind, randomized trial that tested the oral direct factor Xa inhibitor apixaban against aspirin for the prevention of stroke or systemic embolism in individuals with atrial fibrillation and at least one additional risk factor for stroke who were considered unsuitable for vitamin $\mathrm{K}$ antagonist therapy. AVERROES was stopped early due to a superior ratio of benefit to risk of apixaban over aspirin. ${ }^{8}$

At the conclusion of AVERROES, an open-label extension to the study was initiated to allow qualified study participants to receive, or continue to receive, apixaban until the drug became available in their countries, pending regulatory and reimbursement approvals. During the open-label extension, patients were followed on a regular basis, and patient-important outcomes, including stroke or systemic embolism, hemorrhagic stroke, and major bleeding, were recorded. This report describes the long-term efficacy and safety outcomes of patients receiving apixaban during the open-label extension. Further, outcomes of all patients receiving apixaban at any time from the start of the double-blind phase of AVERROES until the end of the open-label extension are reported.

\section{Methods}

In AVERROES, a total of 5,599 patients with atrial fibrillation and at least one additional risk factor for stroke, who were considered unsuitable for vitamin K antagonist therapy, were enrolled at 522 sites in 36 countries between 2007 and 2009. The mean age of participants was 70 years, $58.5 \%$ were male, and the average $\mathrm{CHADS}_{2}$ score was 2.1. Patients were randomized to receive either apixaban $(n=2,808)$ or aspirin $(n=2,791)$. In May 2010, after a mean follow-up duration of 1.1 years, the Data and Safety Monitoring Board recommended early termination of the trial due to a clear benefit in favor of apixaban. Compared with aspirin, apixaban significantly reduced the risk of stroke or systemic embolism (hazard ratio with apixaban, $0.45,95 \%$ confidence interval 0.32-0.62), and showed a similar risk of major bleeding (hazard ratio with apixaban, 1.13, 95\% confidence interval 0.74-1.75).

Two months later, final AVERROES study visits and enrollment of eligible patients in the open-label extension study began. - Supplementary Fig. $\mathbf{S 1}$ (available in the online version) shows the study timeline of AVERROES and transition to the open-label extension.

\section{Eligibility}

All patients who had received either apixaban or aspirin during AVERROES, and who were alive after conclusion of AVERROES and treated at participating centers, were eligible for inclusion in the open-label extension (-Fig. 1). Additionally, to be included, patients must not have permanently or temporarily (for more than 30 days) discontinued study medication at the time they had their last AVERROES study visit. Exclusion criteria for enrollment in the open-label extension were the same as those for entry in AVERROES, and included the presence of conditions other than atrial fibrillation that required chronic anticoagulation, serious bleeding in the last 6 months, or high risk for bleeding (e.g., patients with active peptic ulcer disease, platelet count $<100,000 / \mathrm{mm}^{3}$, hemoglobin $<10 \mathrm{~g} / \mathrm{dL}$, recent stroke within 10 days, and documented hemorrhagic tendencies), severe renal insufficiency (serum creatinine $>2.5 \mathrm{mg} / \mathrm{dL}$ or a calculated creatinine clearance $<25 \mathrm{~mL} / \mathrm{min}$ ), and alanine aminotransferase/aspartate aminotransferase $>2$ times upper limit of normal or a total bilirubin $>1.5$ times upper limit of normal. Further details can be found in the revised study protocol (version 2.0, 30-Apr-2010, CV185-048), which was made available with the original publication of AVERROES. ${ }^{8,9}$

Patients were required to provide signed written informed consent. Approval of local research ethics boards at participating sites was obtained before enrollment into the open-label extension. If patients were not eligible for the open-label extension or they declined participation, they were discharged from AVERROES and standard of care nonstudy medication was resumed. Study visits occurred at 1,6, and 12 months after entry into the open-label extension, and every 6 months thereafter. Patients participated in the open-label extension until apixaban became available in their country. If a patient discontinued apixaban, their follow-up in the open-label extension was discontinued.

\section{Dosage of Apixaban}

Participants received $5 \mathrm{mg}$ twice daily of open-label apixaban, unless they met prespecified criteria to receive the lower dose of $2.5 \mathrm{mg}$ twice daily (at least 2 out of 3 of: age $\geq 80$ years; body weight $\leq 60 \mathrm{~kg}$; serum creatinine $\geq 1.5 \mathrm{mg} / \mathrm{dL}$ or $133 \mu \mathrm{mol} / \mathrm{L}){ }^{8}$

\section{Study Cohorts and Outcomes of Interest}

Two patient cohorts were analyzed (-Fig. 2). The first cohort comprised all patients that were enrolled in the open-label extension, onset for all analyses being the enrollment in the open-label extension. The second cohort consisted of all patients that were exposed to apixaban during AVERROES or the open-label extension, from the start of AVERROES until the end of open-label extension (hereafter referred to as total apixaban exposure cohort), onset for all analyses being either the start of the double-blind phase of AVERROES (for patients initially randomized to apixaban) or the start of the openlabel extension (for patients randomized to aspirin).

The primary efficacy endpoint was a composite of stroke or systemic embolism. The primary safety endpoint was major bleeding, which was defined as clinically overt bleeding accompanied by one or more of the following: a decrease in the 


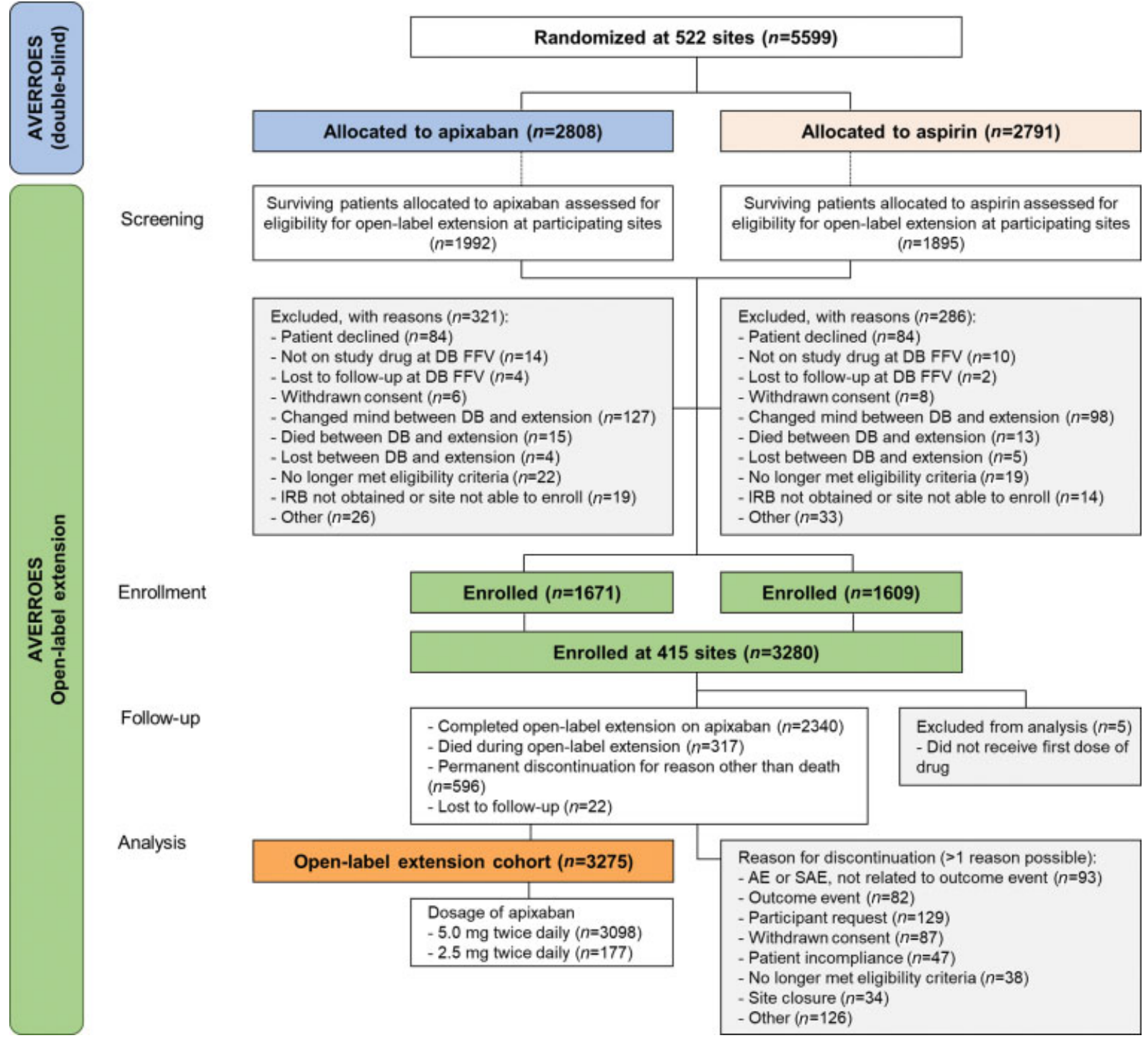

Fig. 1 AVERROES CONSORT diagram. Outcomes of 3,275 patients were analyzed during the open-label extension following conclusion of the double-blind phase. AE, adverse event; DB, double-blind; FFV, final follow-up visit; IRB, institutional review board; SAE, serious adverse event.

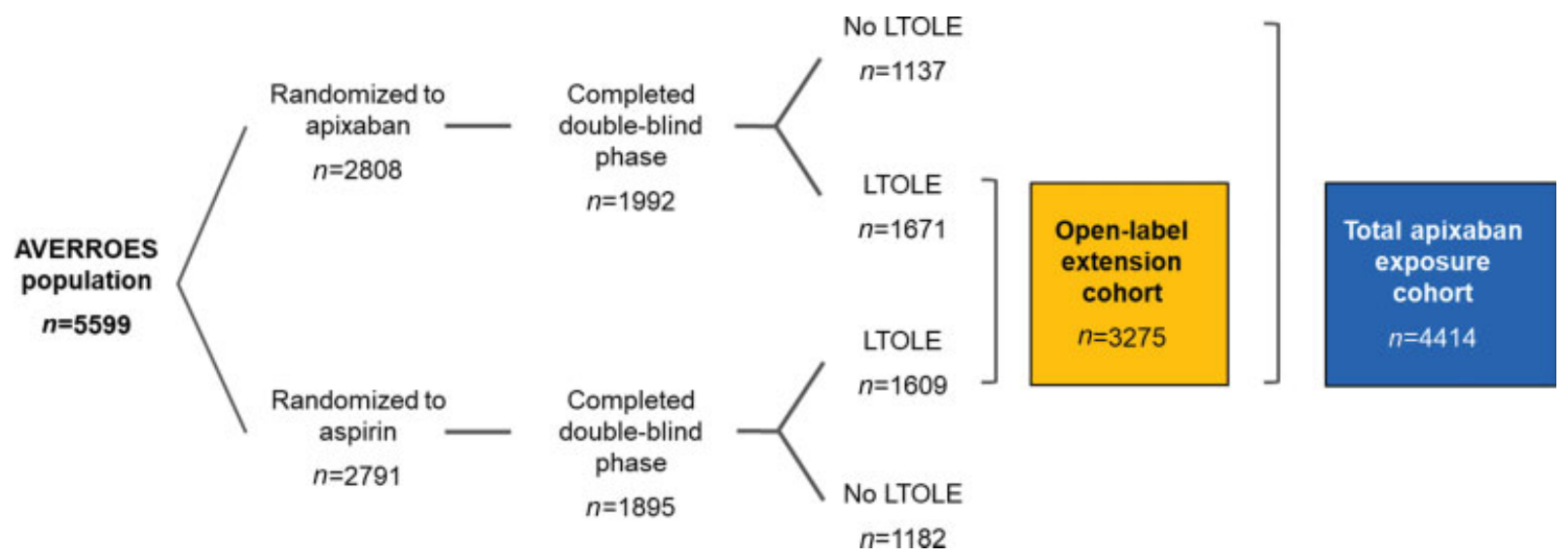

Fig. 2 Composition of the two patient cohorts (open-label extension and total apixaban exposure). A total of 5 patients (out of 3,280) enrolled in the open-label extension did not receive the first dose of apixaban, and were therefore excluded from the analyses. Thus, a total of 3,275 and 4,414 patients were analyzed in the open-label extension and total apixaban exposure cohort, respectively. LTOLE, long-term open-label extension. 
hemoglobin level of $2 \mathrm{~g} / \mathrm{dL}$ or more over a 24-hour period, transfusion of two or more units of packed red cells, bleeding at a critical site (intracranial, intraspinal, intraocular, pericardial, intra-articular, intramuscular with compartment syndrome, or retroperitoneal), or fatal bleeding. Hemorrhagic stroke was a key secondary safety endpoint. Other outcomes of interest were subtype of stroke, systemic embolism, myocardial infarction, clinically relevant nonmajor and minor bleeding, and overall and cardiovascular mortality. Although all efficacy and major bleeding events were adjudicated by an independent committee during the double-blind phase of AVERROES, there was no adjudication of outcome events during the open-label extension.

\section{Data Collection and Statistics}

Baseline characteristics as captured at the onset of the doubleblind phase of AVERROES were reported for patients entering and not entering the open-label extension phase. Fisher's exact test or the chi-square test for categorical variables, depending on the expected cell counts, and the two-sample $t$-test for normally distributed variables or two-sample Wilcoxon test for nonnormally distributed variables were used to test for between-group differences in baseline variables.

Outcomes of interest were reported as total number of events per patient cohort during the time of follow-up, and the yearly incidence (as per 100 patient-years of follow-up) for each outcome was calculated. Selected outcomes (stroke or systemic embolism, hemorrhagic stroke, and major bleeding) were visualized as Kaplan-Meier curves (time-to-first event).

Subgroup analyses according to actual age (captured at entry into the open-label extension), body weight, and renal function (both captured at the onset of AVERROES) were performed. Further, event rates for selected outcomes are shown according to baseline $\mathrm{CHA}_{2} \mathrm{DS}_{2}$-VASc and a modified HAS-BLED score. Additional adjustment to account for imbalances in patient variables between those collected at baseline and those collected at onset of the open-label extension (actual age, previous stroke or transient ischemic attack, hypertension, heart failure or left ventricular ejection fraction of $35 \%$ or less, peripheral artery disease, previous myocardial infarction, diabetes, significant bleeding while on vitamin K antagonist) was performed for the major efficacy and safety outcomes. All data were collected, validated, and analyzed at the Population Health Research Institute at Hamilton Health Sciences and McMaster University, Hamilton, Canada. Statistical analysis was conducted using software SAS (version 9.4).

\section{Study Conduct}

AVERROES and its open-label extension were funded by Bristol-Myers Squibb and Pfizer. The open-label extension was added to the study protocol in April 2010. AVERROES, including a protocol amendment to enable the open-label extension follow-up, is registered at ClinicalTrials.gov (NCT00496769).

\section{Results}

\section{Open-Label Extension Cohort}

Of the 5,599 patients enrolled in AVERROES, a total of 3,280 patients were subsequently enrolled in the open-label extension ( - Fig. 1), 1,671 patients who had received apixaban, and 1,609 individuals who had received aspirin during the double-blind phase. Five patients did not receive the first dose of apixaban. These patients were excluded, leaving a total of 3,275 (58.5\%) patients for analysis. During the openlabel extension, apixaban was approved in many countries

Table 1 Baseline characteristics

\begin{tabular}{|l|l|l|l|}
\hline & $\begin{array}{l}\text { Enrolled in open-label } \\
\text { extension }(N=3,275)\end{array}$ & $\begin{array}{l}\text { Not enrolled in open-label } \\
\text { extension }(N=2,319)\end{array}$ & $p$-Value \\
\hline Age $(y)$, mean \pm SD & $69.2 \pm 9.4$ & $70.8 \pm 9.7$ & $<0.001$ \\
\hline Male sex, $n(\%)$ & $1,928(58.9)$ & $1,344(58.0)$ & 0.506 \\
\hline Heart rate (beats/min), mean \pm SD & $73.4 \pm 14.1$ & $75.2 \pm 14.5$ & $<0.001$ \\
\hline Systolic blood pressure $(\mathrm{mm}$ Hg), mean \pm SD & $131.7 \pm 16.0$ & $131.5 \pm 17.1$ & 0.699 \\
\hline Body mass index $\left(\mathrm{kg} / \mathrm{m}^{2}\right)$, mean \pm SD & $28.5 \pm 5.6$ & $28.1 \pm 5.7$ & 0.010 \\
\hline Risk factors for stroke, $n(\%)$ & & & 0.179 \\
\hline Prior stroke or transient ischemic attack & $431(13.2)$ & $334(14.4)$ & 0.782 \\
\hline Hypertension, receiving treatment & $2,827(86.3)$ & $2,006(86.6)$ & $<0.001$ \\
\hline Heart failure & $1,167(35.6)$ & $1,002(43.2)$ & 0.026 \\
\hline NYHA class I or II & $992(85.0)$ & $816(81.4)$ & 0.026 \\
\hline NYHA class III or IV & $175(15.0)$ & $186(18.6)$ & 0.576 \\
\hline Left ventricular ejection fraction 35\% or below & $164(5.0)$ & $124(5.4)$ & 0.078 \\
\hline Peripheral artery disease & $79(2.4)$ & $74(3.2)$ & 0.057 \\
\hline Diabetes, receiving treatment & $614(18.7)$ & $482(20.8)$ & 0.061 \\
\hline Mitral stenosis & $57(1.7)$ & $57(2.5)$ & $($ Continued) \\
\hline
\end{tabular}


Table 1 (Continued)

\begin{tabular}{|c|c|c|c|}
\hline & $\begin{array}{l}\text { Enrolled in open-label } \\
\text { extension }(N=3,275)\end{array}$ & $\begin{array}{l}\text { Not enrolled in open-label } \\
\text { extension }(N=2,319)\end{array}$ & $p$-Value \\
\hline \multicolumn{4}{|c|}{ Classification of atrial fibrillation, $n(\%)$} \\
\hline Paroxysmal, $n(\%)$ & $919(28.1)$ & $593(25.6)$ & 0.041 \\
\hline Persistent, $n$ (\%) & $715(21.8)$ & $459(19.8)$ & 0.067 \\
\hline Permanent, $n(\%)$ & $1,641(50.1)$ & $1,265(54.6)$ & $<0.001$ \\
\hline \multicolumn{4}{|l|}{$\mathrm{CHADS}_{2}$ score } \\
\hline Mean score \pm SD & $2.0 \pm 1.0$ & $2.2 \pm 1.1$ & $<0.001$ \\
\hline \multicolumn{4}{|l|}{ Score, $n(\%)$} \\
\hline 0 or 1 & 1,307 (39.9) & $716(30.9)$ & $<0.001$ \\
\hline 2 & $1,148(35.1)$ & $850(36.7)$ & 0.205 \\
\hline 3 or more & $820(25.0)$ & $750(32.4)$ & $<0.001$ \\
\hline \multicolumn{4}{|l|}{$\mathrm{CHA}_{2} \mathrm{DS}_{2}$-VASc score ${ }^{\mathrm{a}}$} \\
\hline Mean score \pm SD & $3.3 \pm 1.4$ & $3.4 \pm 1.5$ & $<0.001$ \\
\hline \multicolumn{4}{|l|}{ Score, $n(\%)$} \\
\hline 0 or 1 & $313(9.6)$ & $187(8.1)$ & 0.056 \\
\hline 2 or 3 & $1,628(49.7)$ & $1,083(46.8)$ & 0.030 \\
\hline 4 or more & $1,334(40.7)$ & $1,046(45.2)$ & $<0.001$ \\
\hline \multicolumn{4}{|l|}{ HAS-BLED score ${ }^{\mathrm{b}}$} \\
\hline Mean score \pm SD & $1.1 \pm 0.8$ & $1.2 \pm 0.8$ & 0.014 \\
\hline \multicolumn{4}{|l|}{ Score, $n(\%)$} \\
\hline 0 or 1 & $2,403(73.4)$ & $1,586(68.8)$ & $<0.001$ \\
\hline 2 & $737(22.5)$ & $619(26.8)$ & $<0.001$ \\
\hline 3 or more & $134(4.1)$ & $101(4.4)$ & 0.599 \\
\hline \multicolumn{4}{|c|}{ Medication use at baseline, $n(\%)$} \\
\hline ACE inhibitor or ARB & $2,092(64.1)$ & $1,482(64.2)$ & 0.938 \\
\hline Verapamil or diltiazem & $301(9.2)$ & $198(8.6)$ & 0.406 \\
\hline Beta-blocker & $1,823(55.8)$ & $1,272(55.1)$ & 0.578 \\
\hline Digoxin & $906(27.7)$ & $667(28.9)$ & 0.354 \\
\hline Amiodarone & $342(10.5)$ & $284(12.3)$ & 0.034 \\
\hline Statin & $1,143(35.0)$ & $718(31.1)$ & 0.003 \\
\hline
\end{tabular}

Abbreviations: ACE, angiotensin-converting enzyme; ARB, angiotensin II receptor blocker; NYHA, New York Heart Association; SD, standard deviation.

Note: Baseline characteristics are shown as captured at the time of enrollment into the AVERROES double-blind phase. $p$-Value is from Fisher's exact test or the chi-square test for categorical variables, depending on the expected cell counts, and the two-sample $t$-test for normally distributed variables or two-sample Wilcoxon test for nonnormally distributed variables. Missing data for patients entering the open-label extension: left ventricular ejection fraction $35 \%(n=7)$, HAS-BLED score $(n=1)$, medication use at baseline $(n=9)$; missing data for patients not entering the openlabel extension: sex $(n=1)$, prior stroke or transient ischemic attack, hypertension, heart failure, peripheral artery disease, and diabetes (all $n=2)$, left ventricular ejection fraction $35 \%$ or less $(n=3)$, classification of atrial fibrillation $(n=2), \mathrm{CHADS}_{2}$ score $(n=3), \mathrm{CHA}_{2} \mathrm{DS}_{2}-\mathrm{VASc} \mathrm{score}(n=3)$, HASBLED score $(n=13)$, medication use at baseline except statin use $(n=9)$, and statin use $(n=12)$.

${ }^{\mathrm{a}} \mathrm{The}_{\mathrm{CHA}} \mathrm{DS}_{2}$-VASc score ranges from 0 to 9 (2 points for each age $>75$ years and prior stroke or transient ischemic attack, 1 point for each hypertension, diabetes, vascular disease, age 65 to 74 years, or female sex. Vascular disease was defined as peripheral artery disease or pathological Q wave on electrocardiogram (ECG).

${ }^{\mathrm{b}}$ Due to missing information for some components, a modified HAS-BLED score was used. This score ranges from 0 to 7 ( 1 point for each hypertension [systolic blood pressure $>160 \mathrm{~mm} \mathrm{Hg}$ ], liver disease, prior stroke or transient ischemic attack, significant or major bleeding on vitamin $\mathrm{K}$ antagonist, age $>65$ years, concomitant therapy with an antiplatelet or nonsteroidal anti-inflammatory drug, and 8 or more alcoholic drinks per week). Compared with the original HAS-BLED score (ranging from 0 to 9), every patient received 0 points for renal disease (data needed not available) and labile international normalized ratio (INR) (no patient on vitamin K antagonist during open-label extension). 
which happened in a staggered fashion. Based on the timing of these approvals and commercial availability of apixaban, sites were notified to begin completing final visits and transition patients off of the study medication.

- Table 1 shows the baseline characteristics of patients entering and not entering the open-label extension, data being captured at the onset of AVERROES. There were some significant differences in baseline characteristics of the two groups, including patients entering the open-label extension being younger, being less likely to have a history of heart failure, having a higher proportion of paroxysmal atrial fibrillation, and a lower $\mathrm{CHADS}_{2}$ and $\mathrm{CHA}_{2} \mathrm{DS}_{2}$-VASc score, as compared with those not entering the open-label extension. There were 177 patients in the open-label extension (5.4\%), who received the reduced apixaban dose of $2.5 \mathrm{mg}$ twice daily. Median (interquartile range) follow-up duration in the open-label extension was $3.0(2.5-3.5)$ years.

\section{Efficacy and Safety of Apixaban during the Open-Label Extension}

Primary and secondary efficacy and safety outcomes for the open-label extension cohort are shown in - Table 2. In this cohort ( $n=3,275)$, the yearly rate of the primary composite endpoint of stroke or systemic embolism was $1.0 \%$. Rates for ischemic as well as unspecified stroke were 0.5 and $0.1 \%$, respectively, and systemic embolism occurred at an annual rate of $0.1 \%$. The yearly rate of hemorrhagic stroke was $0.3 \%$. The yearly rate of major bleeding was 1.2\%. - Fig. 3 shows (unadjusted) Kaplan-Meier curves for the composite of stroke or systemic embolism, for hemorrhagic stroke, and for major bleeding. Additional analyses adjusting for imbalances in baseline variables for patients entering and not entering the open-label extension showed similar annual event rates for the composite of stroke or systemic embolism and major bleeding (see - Supplementary Table S1, available in the online version).

\section{Subgroup Analyses}

Event rates of selected outcomes according to baseline $\mathrm{CHA}_{2} \mathrm{DS}_{2}$-VASc and a modified HAS-BLED score during the open-label extension are shown in -Table 3. The corresponding Kaplan-Meier curves are shown in - Fig. 4. Selected outcomes for subgroups according to age, body weight, and renal function during the open-label extension are shown in - Table 4. The corresponding Kaplan-Meier curves are attached in the Supplementary Material (-Supplementary Figs. S2-S4, available in the online version).

Table 2 Outcomes

\begin{tabular}{|c|c|c|c|c|}
\hline & \multicolumn{2}{|c|}{ Open-label extension cohort } & \multicolumn{2}{|c|}{ Total apixaban exposure cohort } \\
\hline Number of patients $(n)$ & \multicolumn{2}{|l|}{3,275} & \multicolumn{2}{|l|}{4,414} \\
\hline Median (interquartile range) follow-up & \multicolumn{2}{|l|}{$3.0(2.5-3.5)$ years } & \multicolumn{2}{|l|}{$3.0(1.6-4.1)$ years } \\
\hline Outcomes & Number of events & Incidence & Number of events & Incidence \\
\hline Stroke or systemic embolism & 98 & 1.0 & 149 & 1.1 \\
\hline Stroke, systemic embolism, or death & 381 & 4.1 & 522 & 4.0 \\
\hline $\begin{array}{l}\text { Stroke, systemic embolism, myocardial infarction, } \\
\text { or death from vascular reason }\end{array}$ & 267 & 2.8 & 398 & 3.1 \\
\hline $\begin{array}{l}\text { Stroke, systemic embolism, myocardial infarction, } \\
\text { or death from vascular reason, or major bleeding }\end{array}$ & 337 & 3.6 & 468 & 3.6 \\
\hline \multicolumn{5}{|l|}{ Stroke } \\
\hline All & 88 & 0.9 & 137 & 1.1 \\
\hline Ischemic & 48 & 0.5 & 83 & 0.6 \\
\hline Hemorrhagic & 29 & 0.3 & 35 & 0.3 \\
\hline Unspecified & 11 & 0.1 & 20 & 0.2 \\
\hline Systemic embolism & 12 & 0.1 & 14 & 0.1 \\
\hline Myocardial infarction & 59 & 0.6 & 83 & 0.6 \\
\hline \multicolumn{5}{|l|}{ Death } \\
\hline From any cause & 317 & 3.4 & 428 & 3.3 \\
\hline From vascular cause & 199 & 2.1 & 283 & 2.2 \\
\hline From nonvascular cause & 118 & 1.3 & 145 & 1.1 \\
\hline \multicolumn{5}{|l|}{ Bleeding } \\
\hline Major & 110 & 1.2 & 153 & 1.2 \\
\hline Clinically relevant non major & 152 & 1.6 & 247 & 1.9 \\
\hline Minor & 259 & 2.9 & 422 & 3.5 \\
\hline
\end{tabular}

Note: Incidence is reported as per 100 patient-years. 

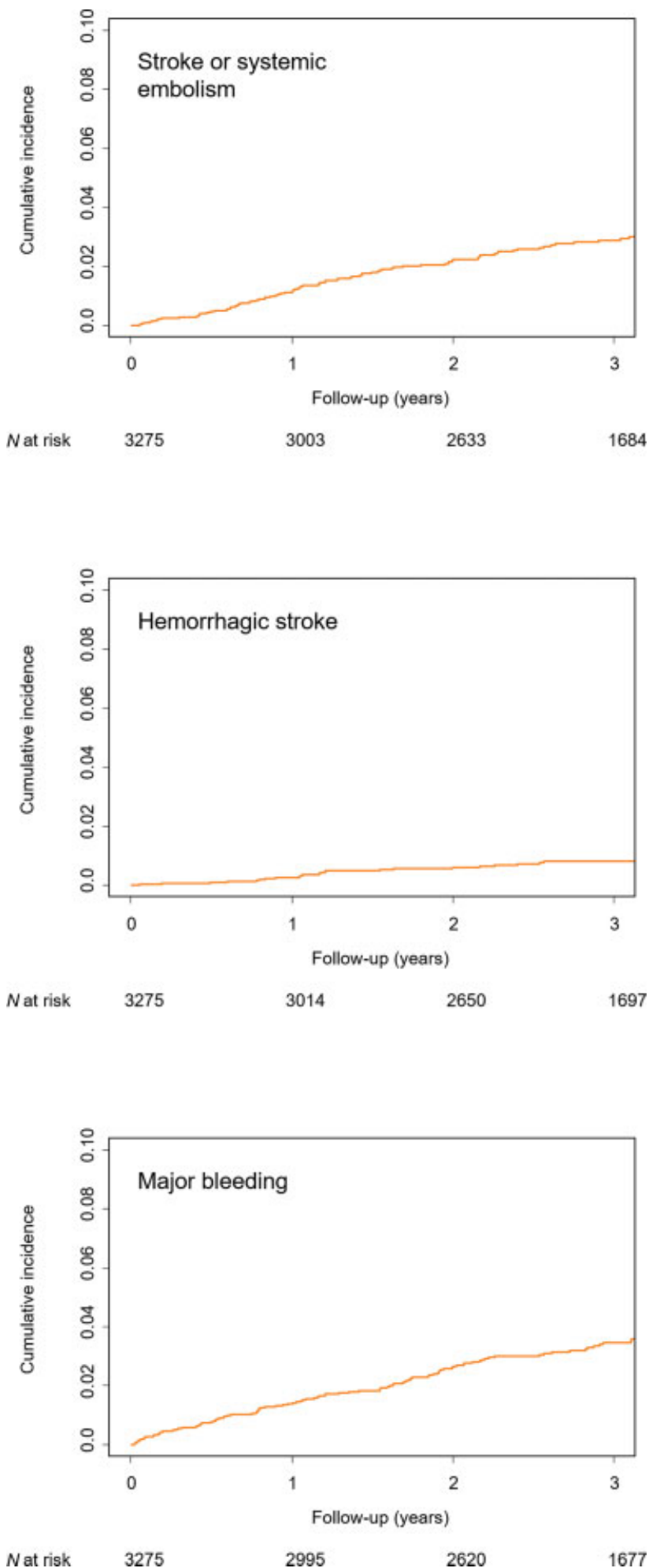

Fig. 3 Unadjusted Kaplan-Meier curves for selected outcomes (openlabel extension cohort). Curves truncated.

Other Outcomes of Interest during and after the OpenLabel Extension

The rate of myocardial infarction was $0.6 \%$ per year for the open-label extension cohort. Yearly rates of death from any cause and cardiovascular death were 3.4 and $2.1 \%$, respectively. Rates of clinically relevant nonmajor and minor bleeding were 1.6 and $2.9 \%$ per year. - Supplementary Table $\mathbf{S 2}$
Table 3 Event rates for selected outcomes during the openlabel extension according to baseline $\mathrm{CHA}_{2} \mathrm{DS}_{2}$-VASc and HASBLED score

\begin{tabular}{|l|l|l|l|}
\hline Outcome & Score & $\begin{array}{l}\text { Events/ } \\
\text { patients }\end{array}$ & Incidence \\
\hline $\begin{array}{l}\text { Stroke or } \\
\text { systemic } \\
\text { embolism }\end{array}$ & $\begin{array}{l}\mathrm{CHA}_{2} \mathrm{DS}_{2} \text {-VASc } \\
\text { score }\end{array}$ & & \\
\cline { 2 - 4 } & 0 or 1 & $5 / 313$ & 0.5 \\
\cline { 2 - 4 } & 2 or 3 & $43 / 1,628$ & 0.9 \\
\cline { 2 - 4 } & 4 or greater & $50 / 1,334$ & 1.4 \\
\hline \multirow{4}{*}{$\begin{array}{l}\text { Hemorrhagic } \\
\text { stroke }\end{array}$} & HAS-BLED score & & \\
\cline { 2 - 4 } & 0 or 1 & $23 / 2,403$ & 0.3 \\
\cline { 2 - 4 } & 2 & $4 / 737$ & 0.2 \\
\cline { 2 - 4 } & 3 or greater & $2 / 134$ & 0.6 \\
\hline \multirow{4}{*}{$\begin{array}{l}\text { Major } \\
\text { bleeding }\end{array}$} & HAS-BLED score & & \\
\cline { 2 - 4 } & 0 or 1 & $81 / 2,403$ & 1.2 \\
\cline { 2 - 4 } & 2 & $19 / 737$ & 0.9 \\
\cline { 2 - 4 } & 3 or greater & $10 / 134$ & 2.9 \\
\hline
\end{tabular}

Note: Incidence is reported as per 100 patient-years.

(available in the online version) shows event rates for patients that had been allocated to apixaban, and for patients that had been allocated to aspirin during the double-blind phase of AVERROES, and there were no significant betweengroup differences in outcomes during the open-label extension. - Supplementary Table $\mathbf{S 3}$ (available in the online version) shows event rates for the 177 patients who were receiving apixaban at a dose of $2.5 \mathrm{mg}$ twice daily.

During a median follow-up duration of 3.0 years, a total of 596 out of 3,275 (18.2\%) patients in the open-label extension cohort discontinued apixaban early (-Fig. 1). Antithrombotic therapy and site-reported clinical outcomes during the first 30 days after completion of the open-label extension are shown in $\boldsymbol{\sim}$ Supplementary Table $\mathbf{5 4}$ (available in the online version).

\section{Total Apixaban Exposure Cohort}

This cohort allowed analyses of all patients that received apixaban, at any time from the beginning of AVERROES until the conclusion of the open-label extension. It comprised 4,414 patients (open-label extension cohort as described previously, and patients allocated to apixaban during AVERROES but not enrolled in the extension). Median (interquartile range) follow-up duration in the total apixaban exposure cohort was 3.0 (1.6-4.1) years. The yearly rate of the composite of stroke or systemic embolism was $1.1 \%$ (-Table 2). Annual rates for ischemic and unspecified stroke, and systemic embolism were $0.6,0.2$, and $0.1 \%$, respectively. The yearly rate of hemorrhagic stroke was $0.3 \%$. The rate of major bleeding was $1.2 \%$ per year. - Fig. 5 shows (unadjusted) Kaplan-Meier curves for the composite of stroke or systemic embolism, for hemorrhagic stroke, and for major bleeding for the total apixaban exposure cohort. 

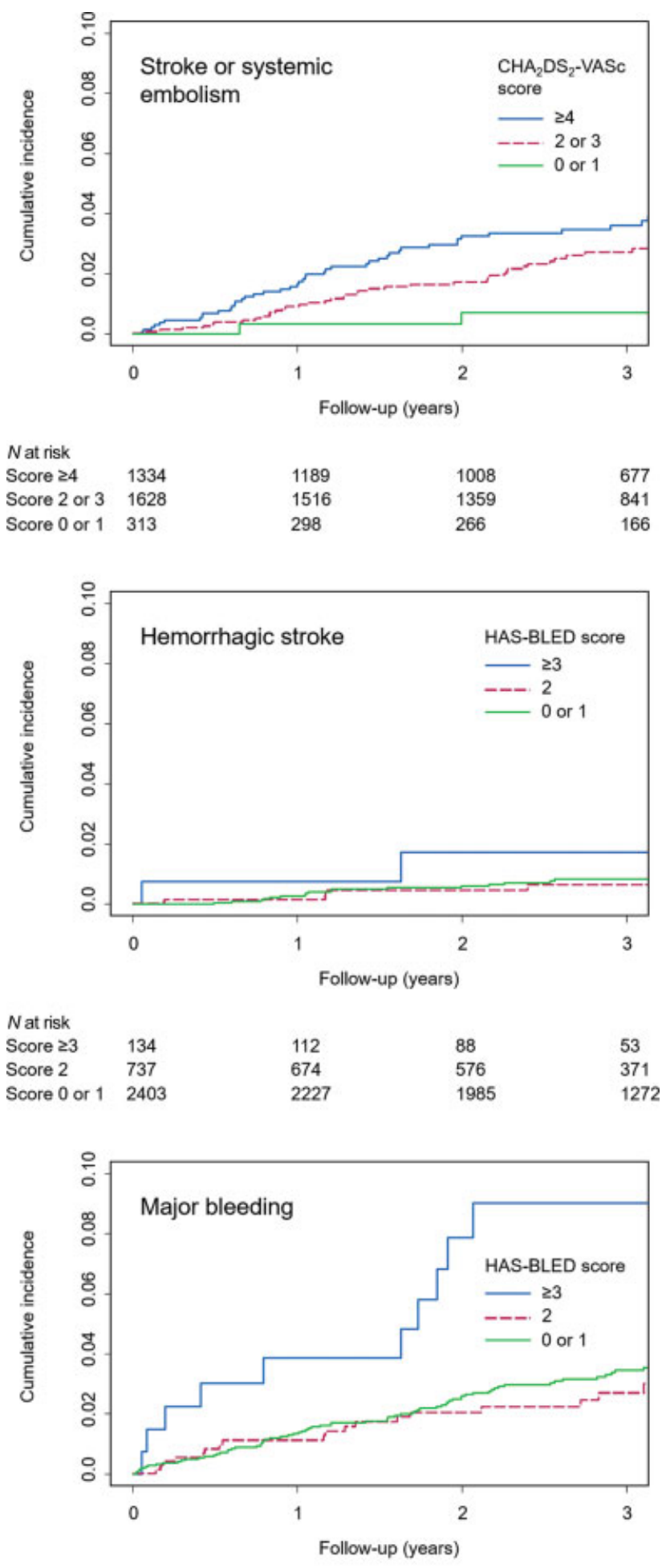

$N$ at risk

$\begin{array}{lllll}\text { Score } \geq 3 & 134 & 111 & 84 & 51 \\ \text { Score } 2 & 737 & 670 & 572 & 368 \\ \text { Score } 0 \text { or } 1 & 2403 & 2213 & 1963 & 1257\end{array}$

Fig. 4 Unadjusted Kaplan-Meier curves for selected outcomes according to $\mathrm{CHA}_{2} \mathrm{DS}_{2}$-VASc and HAS-BLED score (open-label extension-cohort). Curves truncated.

\section{Discussion}

The oral direct factor Xa inhibitor apixaban has been shown to be both effective and safe in patients with atrial fibrillation at
Table 4 Event rates for selected outcomes during the openlabel extension according to age, body weight, and kidney function

\begin{tabular}{|c|c|c|c|c|}
\hline & Outcome & Subgroup & $\begin{array}{l}\text { Events/ } \\
\text { patients }\end{array}$ & Incidence \\
\hline \multirow[t]{6}{*}{ Age (y) } & \multirow{2}{*}{$\begin{array}{l}\text { Stroke or } \\
\text { systemic } \\
\text { embolism }\end{array}$} & $\geq 80$ & $23 / 635$ & 1.4 \\
\hline & & $<80$ & $75 / 2,640$ & 1.0 \\
\hline & \multirow{2}{*}{$\begin{array}{l}\text { Hemorrhagic } \\
\text { stroke }\end{array}$} & $\geq 80$ & $8 / 635$ & 0.5 \\
\hline & & $<80$ & $21 / 2,640$ & 0.3 \\
\hline & \multirow{2}{*}{$\begin{array}{l}\text { Major } \\
\text { bleeding }\end{array}$} & $\geq 80$ & $37 / 635$ & 2.3 \\
\hline & & $<80$ & $73 / 2,640$ & 0.9 \\
\hline \multirow{6}{*}{$\begin{array}{l}\text { Body } \\
\text { weight } \\
\text { (kg) }\end{array}$} & \multirow{2}{*}{$\begin{array}{l}\text { Stroke or } \\
\text { systemic } \\
\text { embolism }\end{array}$} & $\leq 60$ & $17 / 461$ & 1.3 \\
\hline & & $>60$ & $81 / 2,814$ & 1.0 \\
\hline & \multirow{2}{*}{$\begin{array}{l}\text { Hemorrhagic } \\
\text { stroke }\end{array}$} & $\leq 60$ & $7 / 461$ & 0.5 \\
\hline & & $>60$ & $22 / 2,814$ & 0.3 \\
\hline & \multirow{2}{*}{$\begin{array}{l}\text { Major } \\
\text { bleeding }\end{array}$} & $\leq 60$ & $19 / 461$ & 1.4 \\
\hline & & $>60$ & $91 / 2,814$ & 1.1 \\
\hline \multirow{6}{*}{$\begin{array}{l}\text { Kidney } \\
\text { function } \\
\text { (serum } \\
\text { creatinine } \\
\text { in } \mathrm{mg} / \mathrm{dL} \text { ) }\end{array}$} & \multirow{2}{*}{$\begin{array}{l}\text { Stroke or } \\
\text { systemic } \\
\text { embolism }\end{array}$} & $\geq 1.5$ & $10 / 186$ & 2.2 \\
\hline & & $<1.5$ & $88 / 3,089$ & 1.0 \\
\hline & \multirow{2}{*}{$\begin{array}{l}\text { Hemorrhagic } \\
\text { stroke }\end{array}$} & $\geq 1.5$ & $6 / 186$ & 1.3 \\
\hline & & $<1.5$ & $23 / 3,089$ & 0.3 \\
\hline & \multirow{2}{*}{$\begin{array}{l}\text { Major } \\
\text { bleeding }\end{array}$} & $\geq 1.5$ & $12 / 186$ & 2.6 \\
\hline & & $<1.5$ & $98 / 3,089$ & 1.1 \\
\hline
\end{tabular}

Note: Incidence is reported as per 100 patient-years.

risk for thromboembolism. ${ }^{8,10}$ As a result, it is currently being recommended for this indication by American and European guidelines for the management of atrial fibrillation. ${ }^{11,12}$ AVERROES showed a clear benefit for apixaban over aspirin in patients with atrial fibrillation that were considered unsuitable for vitamin K antagonist therapy. ${ }^{8}$ During the open-label extension of AVERROES, apixaban proved to be both effective, with continuing low rates for stroke or systemic embolism, and safe, with low rates for hemorrhagic stroke and major bleeding over a median follow-up duration of 3.0 years. No additional long-term safety concerns were identified.

\section{Efficacy}

The primary composite efficacy endpoint of stroke or systemic embolism tended to be lower in the open-label extension cohort as compared with the yearly event rate in patients allocated to apixaban during AVERROES (1.0 vs. $1.6 \%$ per year) (-Supplementary Table S5, available in the online version). The annual rate of stroke or systemic embolism during the open-label extension is also in line with findings from the ARISTOTLE trial, which tested apixaban against warfarin for the prevention of thromboembolism in patients with atrial fibrillation and at least one risk factor for stroke and found a rate of $1.3 \%$ per year for patients on apixaban. ${ }^{10}$

Additional analyses according to age, body weight, renal function, baseline $\mathrm{CHA}_{S} \mathrm{DS}_{2}$-VASc, and a modified HAS-BLED score demonstrate the efficacy of apixaban across clinically important subgroups of patients with atrial fibrillation. 

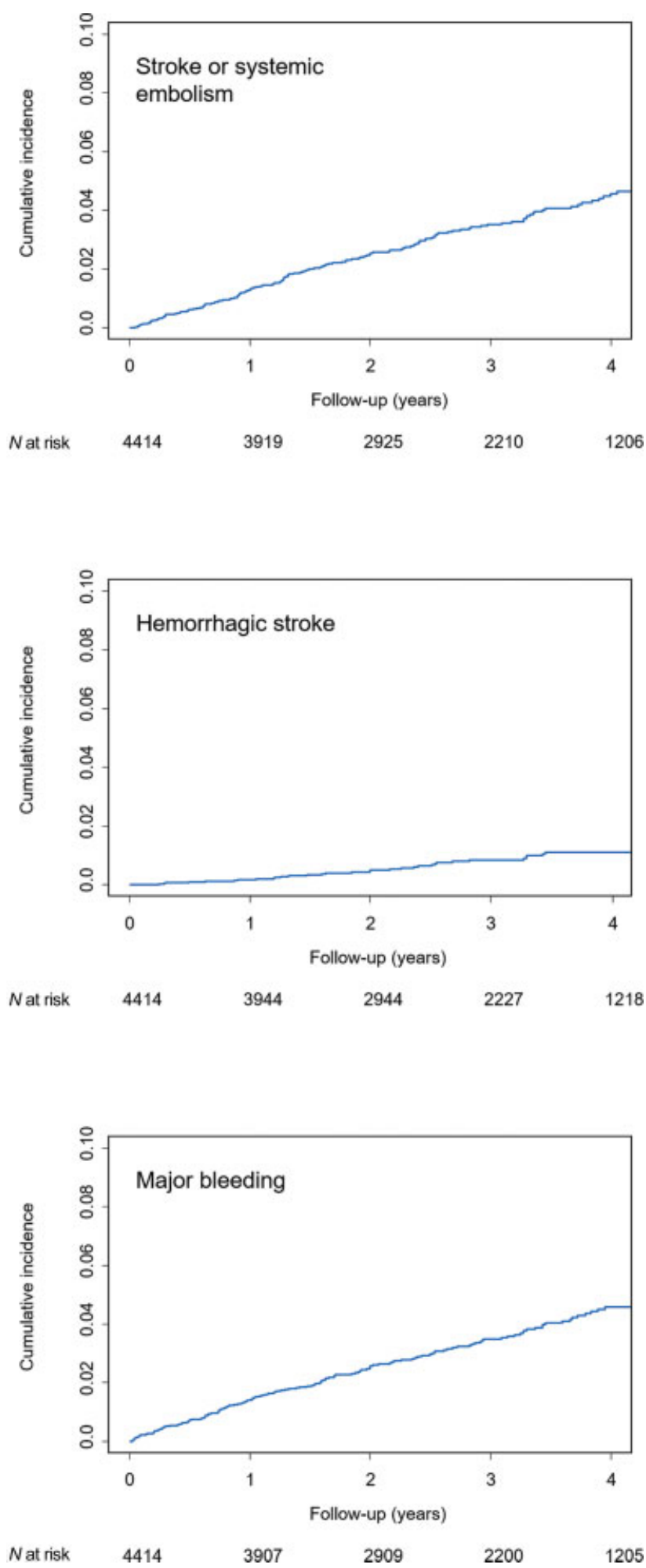

Fig. 5 Unadjusted Kaplan-Meier curves for selected outcomes (total apixaban exposure cohort). Curves truncated.

Our analyses in all patients that received apixaban at any time from the beginning of AVERROES to the end of the openlabel extension (total apixaban exposure cohort) showed that yearly rates for the composite of stroke or systemic embolism are similarly low in this cohort.

\section{Safety}

During the open-label extension, apixaban was shown to be associated with continuing low rates for both hemorrhagic stroke and major bleeding ( 0.3 and $1.2 \%$ per year, respectively), as compared with 0.2 and $1.4 \%$ per year for patients allocated to apixaban during the double-blind phase of AVERROES (-Supplementary Table S5, available in the online version). ${ }^{8}$ These results add to findings derived from the ARISTOTLE study, where yearly rates for hemorrhagic stroke and major bleeding (according to the International Society on Thrombosis and Haemostasis criteria) in study participants allocated to apixaban were 0.2 and $2.1 \%{ }^{10}$

Subgroup analyses provided further insights into the safety profile of apixaban across clinically important subsets of patients during the open-label extension.

In the total apixaban exposure cohort, yearly rates for hemorrhagic stroke and major bleeding tended to be as low as or lower ( 0.3 and $1.2 \%$, respectively) than those observed during apixaban treatment in the double-blind phase of AVERROES.

\section{Other Outcomes of Interest}

During the open-label extension, apixaban was shown to be associated with rates of overall and cardiovascular mortality were similar to those observed during the double-blind phase of the trial, reflecting an older, high-risk patient population with many comorbidities. There was no difference in outcomes during the open-label extension with respect to what antithrombotic regimen patients had been allocated to during the double-blind phase of AVERROES.

\section{Event Rates Compared with Those at the End of Other Anticoagulation Trials}

With respect to other pivotal trials of nonvitamin Kantagonists in patients with atrial fibrillation, apixaban is the only direct oral anticoagulant which has been studied against both aspirin and warfarin, 8,10 whereas the remainder were exclusively tested against vitamin K antagonists (i.e., warfarin). This report showed continuing low rates for important efficacy and safety outcomes after the end of the double-blind phase of AVERROES. Increased rates for both thromboembolic and bleeding events have been observed after conclusion of ARISTOTLE (apixaban) and ROCKET-AF (rivaroxaban), as both studies did not provide an open-label extension and all patients were switched to warfarin after trial conclusion. ${ }^{13,14}$ With respect to edoxaban, ENGAGE AF-TIMI 48 being conducted later in time, a specific transition plan was performed and effectively protected study participants from an excess of thrombotic and bleeding events. ${ }^{15}$ The RELY-ABLE study, which was initiated after the conclusion of RE-LY, provided access to dabigatran only for the patients that had been allocated to receive dabigatran during the double-blind phase of the trial, and showed slightly higher or similar event rates for both efficacy and safety outcomes.

\section{Study Limitations}

Only 58.5\% of participants enrolled in AVERROES entered the open-label extension, which may have influenced the results 
through selection and survivorship bias. The overall risk profile of the subset of patients entering the open-label extension was lower than that of those enrolled in the main trial. More than a third of patients (39.9\%) had a CHADS 2 score of 0 or 1 , indicating low or moderate stroke risk. As opposed to the double-blind phase of the study, there was no adjudication of efficacy and safety outcomes in the open-label extension. We cannot exclude the possibility of underreporting of events. Further, this report is primarily descriptive as there was no control group during the open-label extension. Finally, median follow-up duration in the open-label extension was only 3.0 years and depended on the timing of regulatory and reimbursement approvals.

\section{Conclusion}

Following the completion of the double-blind phase of AVERROES, annual rates of stroke or systemic embolism, hemorrhagic stroke, and major bleeding remained as low as those observed during apixaban treatment in the main trial. These data, based on a median follow-up duration of 3.0 years, support the long-term efficacy and safety of apixaban in patients with atrial fibrillation.

\section{What is known about this topic?}

- The direct oral factor Xa inhibitor apixaban is effective in reducing thromboembolic risk in patients with atrial fibrillation and risk factors for stroke.

- AVERROES was a randomized clinical trial that showed superiority of apixaban over aspirin in atrial fibrillation patients that were considered unsuitable for vitamin $\mathrm{K}$ antagonist therapy.

- At the conclusion of AVERROES, an open-label extension was initiated to allow study participants to receive, or continue to receive, apixaban until it became locally available in their countries.

\section{What does this paper add?}

- During the open-label extension of AVERROES, low annual rates of stroke or systemic embolism (1.0\%), hemorrhagic stroke $(0.3 \%)$, and major bleeding $(1.2 \%)$ were observed in 3,275 patients with atrial fibrillation receiving apixaban over a median follow-up duration of 3.0 years.

- In an analysis of total patient-years exposed to apixaban, from the start of the double-blind phase of AVERROES until the end of the open-label extension, annual event rates were $1.1 \%$ for stroke or systemic embolism, $0.3 \%$ for hemorrhagic stroke, and $1.2 \%$ for major bleeding.

Conflict of Interest

A.P.B. reports travel support to attend a scientific meeting from St. Jude Medical/Abbott, outside the submitted work. J.W.E. reports grants and personal fees from Bristol-Myers Squibb/Pfizer, during the conduct of the study; grants and personal fees from Astra Zeneca, grants and personal fees from Bayer, grants and personal fees from Boehringer Ingelheim, grants and personal fees from Bristol-Myers Squibb/Pfizer, grants and personal fees from Daiichi Sankyo, grants and personal fees from Eli Lilly, grants and personal fees from Glaxo Smith Kline, grants and personal fees from Janssen, grants and personal fees from Sanofi Aventis, outside the submitted work. S. Y. reports grants from Bristol-Myers Squibb/Pfizer, during the conduct of the study; grants and personal fees from Bayer, grants and personal fees from Boehringer Ingelheim, outside the submitted work. S.H.H. reports personal fees from Bristol-Myers Squibb/Pfizer, during the conduct of the study; personal fees from Bayer Healthcare, personal fees from Boehringer Ingelheim, personal fees from Daiichi Sankyo, personal fees from Medtronic, personal fees from St. Jude Medical/Abbott, personal fees from Zoll, outside the submitted work. A.K. is an employee of BristolMyers Squibb. H.B. has nothing to disclose. K.B. has nothing to disclose. J.S.H. reports grants and personal fees from Bristol-Myers Squibb/Pfizer, grants and personal fees from Medtronic, grants and personal fees from Boston Scientific, personal fees from Servier, outside the submitted work. S.J.C. reports grants and personal fees from Bristol-Myers Squibb/Pfizer, during the conduct of the study; grants and personal fees from Portola Pharmaceuticals, grants and personal fees from Bristol-Myers Squibb/Pfizer, grants and personal fees from Bayer, grants and personal fees from Daiichi Sankyo, grants and personal fees from Boehringer Ingelheim outside the submitted work. Open Access support was provided by the Apixaban, Bristol Myers Squibb and Pfizer Alliance.

\section{Funding}

AVERROES and its open-label extension were funded by Bristol-Myers Squibb and Pfizer. A.P.B. reports a personal research grant from the German Heart Foundation (Deutsche Herzstiftung e.V.).

\section{References}

1 Chugh SS, Havmoeller R, Narayanan K, et al. Worldwide epidemiology of atrial fibrillation: a Global Burden of Disease 2010 Study. Circulation 2014;129(08):837-847

2 Go AS, Hylek EM, Phillips KA, et al. Prevalence of diagnosed atrial fibrillation in adults: national implications for rhythm management and stroke prevention: the AnTicoagulation and Risk Factors in Atrial Fibrillation (ATRIA) study. JAMA 2001;285(18):23702375

3 Wolf PA, Abbott RD, Kannel WB. Atrial fibrillation as an independent risk factor for stroke: the Framingham Study. Stroke 1991;22 (08):983-988

4 Healey JS, Oldgren J, Ezekowitz M, et al; RE-LY Atrial Fibrillation Registry and Cohort Study Investigators. Occurrence of death and stroke in patients in 47 countries 1 year after presenting with atrial fibrillation: a cohort study. Lancet 2016;388 (10050):1161-1169 
5 Hart RG, Pearce LA, Aguilar MI. Meta-analysis: antithrombotic therapy to prevent stroke in patients who have nonvalvular atrial fibrillation. Ann Intern Med 2007;146(12):857-867

6 Ruff CT, Giugliano RP, Braunwald E, et al. Comparison of the efficacy and safety of new oral anticoagulants with warfarin in patients with atrial fibrillation: a meta-analysis of randomised trials. Lancet 2014;383(9921):955-962

7 Hohnloser SH, Basic E, Hohmann C, Nabauer M. Effectiveness and safety of non-vitamin $\mathrm{K}$ oral anticoagulants in comparison to phenprocoumon: data from 61,000 patients with atrial fibrillation. Thromb Haemost 2018;118(03):526-538

8 Connolly SJ, Eikelboom J, Joyner C, et al; AVERROES Steering Committee and Investigators. Apixaban in patients with atrial fibrillation. N Engl J Med 2011;364(09):806-817

9 Eikelboom JW, O'Donnell M, Yusuf S, et al. Rationale and design of AVERROES: apixaban versus acetylsalicylic acid to prevent stroke in atrial fibrillation patients who have failed or are unsuitable for vitamin K antagonist treatment. Am Heart J 2010;159(03):348-353.e1

10 Granger CB, Alexander JH, McMurray JJ, et al; ARISTOTLE Committees and Investigators. Apixaban versus warfarin in patients with atrial fibrillation. N Engl J Med 2011;365(11): 981-992
11 January CT, Wann LS, Alpert JS, et al; American College of Cardiology/American Heart Association Task Force on Practice Guidelines. 2014 AHA/ACC/HRS guideline for the management of patients with atrial fibrillation: a report of the American College of Cardiology/American Heart Association Task Force on Practice Guidelines and the Heart Rhythm Society. J Am Coll Cardiol 2014; 64(21):e1-e76

12 Kirchhof P, Benussi S, Kotecha D, et al; ESC Scientific Document Group. 2016 ESC Guidelines for the management of atrial fibrillation developed in collaboration with EACTS. Eur Heart J 2016;37 (38):2893-2962

13 Granger CB, Lopes RD, Hanna M, et al. Clinical events after transitioning from apixaban versus warfarin to warfarin at the end of the Apixaban for Reduction in Stroke and Other Thromboembolic Events in Atrial Fibrillation (ARISTOTLE) trial. Am Heart J 2015;169(01):25-30

14 Mahaffey KW, Hellkamp AS, Patel MR, et al. End of study transition from study drug to open-label vitamin K antagonist therapy: the ROCKET AF experience. Circ Cardiovasc Qual Outcomes 2013; 6(04):470-478

15 Ruff CT, Giugliano RP, Braunwald E, et al. Transition of patients from blinded study drug to open-label anticoagulation: the ENGAGE AF-TIMI 48 trial. J Am Coll Cardiol 2014;64(06):576-584 